\title{
Radio Frequency Identification Sensing Chipless Tag for Permittivity Monitoring of Specific Sizes Materials
}

\author{
Soumaya SAKOUHI ${ }^{1}$, Hedi RAGGAD ${ }^{1}$, Ali GHARSALLAH ${ }^{1}$, Mohamed LATRACH ${ }^{2}$ \\ ${ }^{1}$ Dept. of Physics, Faculty of Sciences of Tunis, University of Tunis El Manar, 2092 Tunis, Tunisia \\ ${ }^{2}$ RF-EMC Research Group, ESEO-IETR, CS90717- 49107, Angers Cedex 2, France \\ soumaya.sakouhi@fst.utm.tn
}

Submitted January 7, 2021 / Accepted June 17, 2021

\begin{abstract}
In this paper, a novel radio frequency identification chipless tag for permittivity sensing characterized by a reduced size, an original shape and a low manufacturing cost is presented. The tag consists of a linear shape taken with multiple linear slots etched on the metal patch, ensuring a multi-frequency response. It enables the development of a robust tag with 8 bits as data capacity within a reduced surface is of $17.5 \times 23 \mathrm{~mm}^{2}$. Hence, using the frequency domain approach, the chipless tag is able to obtain more than 64 different binary states, by the utilization of the frequency shifting technique and the bandwidth distribution. Also, the operating frequency band ranges from 3.5 to $6.5 \mathrm{GHz}$. The new design is simulated, realized and experimentally validated by a bi-static measurement in the anechoic chamber. Then, preliminary tests are used for defining the Radio Frequency sensing chipless tag for permittivity monitoring, and proving its feasibility to control the evolution of a material over time or after use.
\end{abstract}

\section{Keywords}

Radio Frequency Identification (RFID), Frequency Domain Approach (FDA), Ultra Wide Band (UWB), Electro-Magnetic Signal (EMS), permittivity sensor

\section{Introduction}

In wireless identification technologies, the design of small and easily printable structures with relatively low manufacturing costs is the main objective that guarantees its commercialization. The research on RFID chipless technology, which is steadily developed, is an example which takes into account these main challenges, i.e. the cost and printability of the tag. Hence, the chipless RFID tag introduced with success till 2002 in order to overcome the limitations of the conventional RFID [1]. The first proposed prototype is a Surface Acoustic Wave (SAW) based chipless tag which uses a digital modulation based on simultaneous time position and phase shifting at $2.45 \mathrm{GHz}$ ISM band using a series of acoustic reflector taps representing the SAW tag. Its data capacity is 64-bit, which can reach 256 data bits [2], [3]. So, it encodes a large amount of data in a reduced surface. However, the piezoelectric substrate is characterized by a relatively high cost. So, the conventional low-k substrate is the best commercial solution. The RFID chipless tag in this case consists of a linear structure using a classic substrate and embedding the information of identification in the EMS backscattered to the reader [4], [5]. Hence, the choice of the tag shape and the used technique are the dependant parameters necessary to obtain a unique response for each chipless tag. In the last decade, RFID chipless tag was widely studied, and each proposed prototype contributed to the improvement of this technology, and the facilitation of its future industrial propagation. There are essentially two encoding approaches in the chipless technology. The Frequency Domain approach (FDA) is characterized by a short-range identification and an important coding efficiency according to the particular shape [6]. The time Domain approach (TDA) controls the presence and the position of signals reflection in response to a short interrogation pulse. Also, it is characterized by an important read range, but a limited coding efficiency [7]. A number of printable prototypes have been reported using different technologies [8], [9]. The chipless tag presented in [8] describes an array of MEMS switching modules defined by their pull in voltages which is designed to be turned on for a particular time slot, in order to distribute 3 bits of information. The chipless tag proposed in [9] uses the time and frequency domain responses to ensure 3 data bits as an encoding capacity. It also uses short-time matrix pencil method (STMPM) and short-frequency matrix pencil method (SFMPM) to generate the ID by means of size-reduced chipless tag characterized by an elliptical shape and loaded with three slots. In [10], a chipless tag encodes data using a space angle between two V-shaped arms and is identified by measuring the scattered field in two orthogonal polarization directions giving 3 data bits.

The SIW technology is used in [11] as a resonant cavity associated to UWB bowtie antenna forming a chipless tag characterized by low losses and an important quality factor. A chipless tag with multifrequency response is presented in [12], it consists of a microstrip grounded structure based on a circular patch coupled with meandered 
metal strip. The adopted encoding frequency approach ensures a capacity of 20 data bits in the ultra-wideband. In [13], a 9-bit data dense chipless RFID tag consisting of nine vertically polarized slots etched in the metal plate was proposed. The novelty of the proposed tags lies on the use of flexible substrates i.e. Polyethylene terephthalate (PET) and Kapton. The slots are etched in the radiating metal portion of the tag to obtain nine resonances.

Recently, various applications have been already suggested for chipless technology, such as integrating a sensor function with chipless RFID tag [14-18]. The work presented in [14] describes a fully wireless sensory system where a chipless strategy is followed in the sensor part which consists of an LC circuit manufactured by printing techniques on a flexible substrate, whose electrical permittivity shows dependence with the moisture content. Similarly, a compact sensor for the detection of liquid material properties was designed in [15]. A low-cost chipless RFID sensor tag is presented in [16] to determine the dielectric properties of materials. The sensor is based on a depolarizing dipole resonator loaded with printed capacitors whose capacitance depends on the permittivity of the material located on contact with the tag. In [17], the design of an improved radio frequency identification chipless tag-based humidity sensor is proposed. It is characterized by a reduced size, low losses, and high-quality factor. This work evaluates the ability of the tag to be used as a passive sensor detecting the humidity variation by means of a very sensitive material to the humidity. As well, a multi-resonance chipless RFID sensor tag for characterization of defects such as crack and corrosion on metal is presented in [18], it is composed of crossed diagonal dipole patches and L-shaped patches generating six resonance peaks within 2 to $6 \mathrm{GHz}$ band.

For this reason, a novel compact slotted hand-loaded RFID chipless tag for permittivity monitoring is presented in this paper in order to contribute to the improvement of chipless technology in terms of size, encoding capacity, and encoding approach. The proposed design is an improvement of the previous work presented in [19], by using the encoding surface as a whole tag for communication with the reader.

Section 2 describes the working principle and behavior of our tag and explains its way of radiation. The overall design is demonstrated in Sec. 3, and applied in Sec. 4 as a chipless permittivity sensor bringing a real improvement in the chipless technology.

\section{RFID Chipless Tag Study}

\subsection{Slots-loaded RFID Chipless Tag Presentation}

The proposed chipless design consists of a linear structure which is mainly a grounded rectangular form based on a hand-shaped patch and characterized by 4

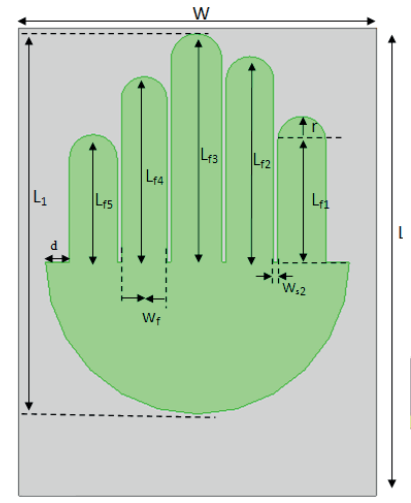

(A)
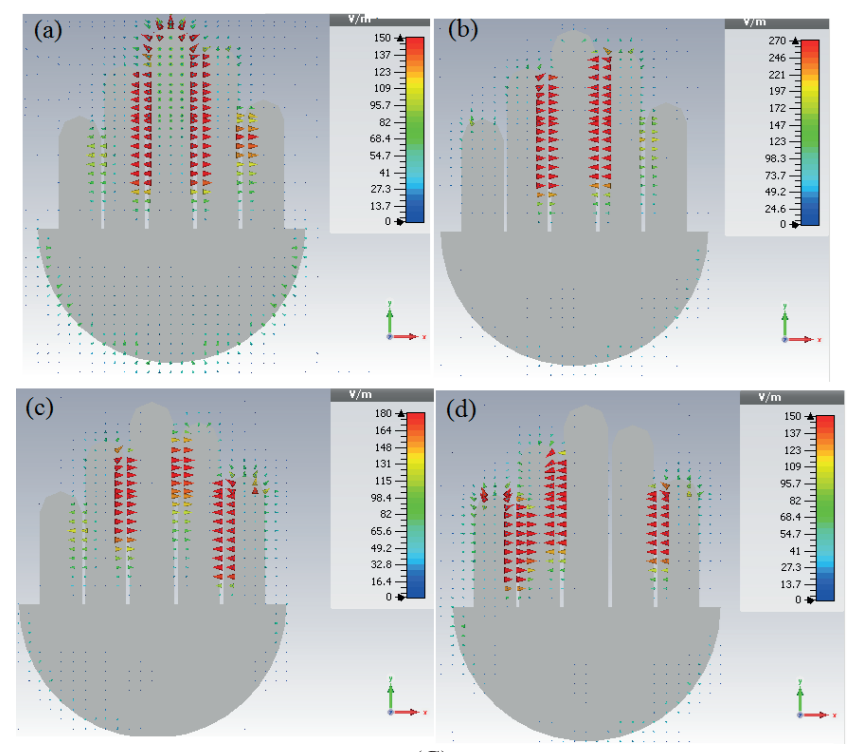

(C)

Fig. 1. (A) The parameters of the slotted hand based chipless tag. (B) The permittivity sensor-based RFID chipless tag. (C) The way of radiation of the proposed chipless tag at (a) $=F 1$, at (b) $=F 2,(\mathrm{c})=F 3$, and (d) $=F 4$.

\begin{tabular}{|c|c|c|c|}
\hline Parameter & Value $(\mathbf{m m})$ & Parameter & Value $(\mathbf{m m})$ \\
\hline $\boldsymbol{W}$ & 17.5 & $\boldsymbol{L}_{\mathrm{f} 4}$ & 9.125 \\
\hline $\boldsymbol{L}$ & 23 & $\boldsymbol{L}_{\mathrm{f} 5}$ & 6.275 \\
\hline $\boldsymbol{L}_{\mathbf{1}}$ & 18.72 & $\boldsymbol{r}$ & 1.175 \\
\hline $\boldsymbol{L}_{\mathrm{f} 1}$ & 6 & $\boldsymbol{W}_{\mathrm{s} \mathbf{2}}$ & 0.2 \\
\hline $\boldsymbol{L}_{\mathrm{f} 2}$ & 10.125 & $\boldsymbol{W}_{\mathrm{f}}$ & 2.5 \\
\hline $\boldsymbol{L}_{\mathrm{f} 3}$ & 11.125 & $\boldsymbol{d}$ & 1.17 \\
\hline
\end{tabular}

Tab. 1. Dimensions of the slotted hand based chipless tag

etched slots on the upper of a hand-shaped metal side. It is characterized by a compact size, and an original shape. Hence, its geometry is expected ensuring a multi-frequency response, mainly more than 64 different binary states, by the utilization of the frequency shifting technique and the bandwidth distribution.

Figure 1(A) shows the proposed slotted hand-based chipless tag. The cutting slots in the top of the metal structure (patch) lead to reduce the size and improve the responses. Furthermore, the generation of the EMS is ensured by changing the parameters in the tag in order to obtain an ID (Identifier) in the reader side. The dimension 
of each parameter is detailed in Tab. 1. It is a design where the four slots are opened from the top side of the hand, and each finger has an optimized dimension in order to ensure the required result. Also, the existence of the ground plane makes the whole chipless tag robust to the material influence in some specific applications of identification, especially when printed on paper, plastic or human body [20].

We need to mention that the first slot length is $L_{\mathrm{f} 1}=$ $6 \mathrm{~mm}$, the second slot length $L_{\mathrm{s} 2}$ is obtained when subtracting the finger length $L_{\mathrm{f} 2}$ by the $r$ dimension of the upper side of the finger. With the same principle, the third slot length and the fourth slot lengths are obtained, appointed respectively $L_{\mathrm{S} 3}$ and $L_{\mathrm{S} 4}$.

\subsection{Working Principle: Discussion}

The principle behind the operation of the proposed chipless tag based on a slotted hand is shown in Fig. 2. The tag is interrogated with a linearly polarized transmitter antenna $\left(T_{x}\right)$. Based on the selected frequency band and the encoding approach, and according to the slots position and dimensions, the encoded frequency signal is backscattered from the tag and received by a linearly polarized receiver antenna $\left(R_{x}\right)$. Thus, the identification task is achieved.

The idea is to find a chain of electromagnetic signatures in order to give an ID for each resonant peak obtained with the previously principle described. In our case, the existing chain is normally based on the slots etched on the hand patch, and the frequency encoding approach can be adopted. In fact, the proposed response is the Radar Cross Section (RCS) representation based on the interrogation signal came from the reader. Thus, the basic description of RCS is the ratio of the scattered power to the incident power in the direction of the observer [21].

\section{RFID Chipless Tag Validation}

\subsection{Design and Results}

Figure 1(A) illustrates the geometry of the proposed RFID chipless tag design, where $L$ and $W$ refer to the tag length of $23 \mathrm{~mm}$ and its width of $17.56 \mathrm{~mm}$ respectively. The used substrate is FR4, with a thickness of $0.8 \mathrm{~mm}$, a dielectric permittivity of 4.6 , and a loss tangent of 0.025 .

The simulation and optimization procedure are achieved using CST Microwave Studio. The slotted hand tag was illuminated with an incident plane wave and a probe placed around with a distance of $200 \mathrm{~mm}$ along $\mathrm{Z}$ axis. Figure 5 shows the simulation result which ensures a multi-frequency response with 4 resonant peaks, namely $F_{1}=3.7 \mathrm{GHz}, F_{2}=4.46 \mathrm{GHz}, F_{3}=5 \mathrm{GHz}, F_{4}=5.75 \mathrm{GHz}$. Thus, data is encoded using the sub band of UWB, i.e., from 3.5 to $6.5 \mathrm{GHz}$, by means of four etched slots.

Figure 1(C) demonstrates the E-field distribution of the chipless tag for each resonant frequency. The E field

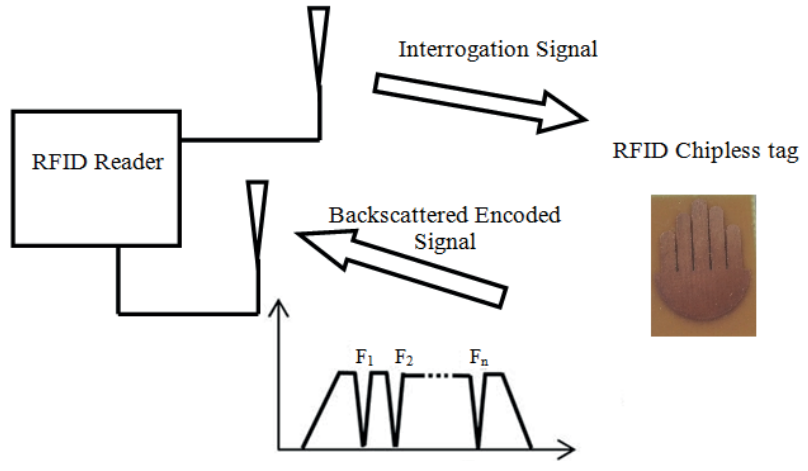

Fig. 2. Working principle of the proposed tag.

distributions at the four frequencies provide the information about the way of the tag radiates, mainly the effect of the slots on each frequency. Hence, the E-field distribution of our hand-shaped tag at the first frequency does not dominate over the structure. But we can find that it is concentrated extensively within the first three slots. Also, the Efield distribution at $F_{2}$ is concentrated within the second and the third slot. Figure 1(C) shows at (c) that the maximum concentration of its distribution at $F_{3}$ is located in the first and third slot and with a minimum radiation in the second slot. Further, the distribution at $F_{4}$ is ensured by the first, third and fourth slot.

Accordingly, we can theoretically derive and predict the frequency by means of the slots dependent on its existence, using the E-field distribution shown in Fig. 1(C) at (a), (b), (c) and (d), and the formula presented in [20], as follows:

$$
f_{\mathrm{r}}=\frac{c}{2 l} \sqrt{\frac{2}{1+\varepsilon_{\mathrm{r}}}}
$$

where $c$ is the speed of light, $\varepsilon_{\mathrm{r}}$ is the relative permittivity of the substrate, and $l$ is the length of slot.

It is needed to mention that to study the way of radiation of the slotted hand-shaped chipless tag for each frequency, a specific formula of length has to be obtained to predict each frequency separately because its existence depends essentially on the slots having the major effect mainly the maximum E-field distribution. Given that $l$ can be one of the slots lengths detailed in Tab. 1 or the sum of the overall length, it depends on the slots affecting each frequency appearance.

Thereby, the experimental investigation aimed to validate the simulation results and to demonstrate the feasibility of our proposed design. Hence, the configuration is prototyped and measured. Figure 3 shows the realized prototype which was fabricated using the standard FR4 substrate. It has a reduced size, allowing it to be used in different applications.

The measurement of the RFID chipless tag is achieved in good conditions in anechoic chamber. The prototype was tested using a bi-static measurement set-up (see Fig. 4) which consists of the Network Analyzer (VNA) Agilent technology N5247 PNA-X covering the frequency range 


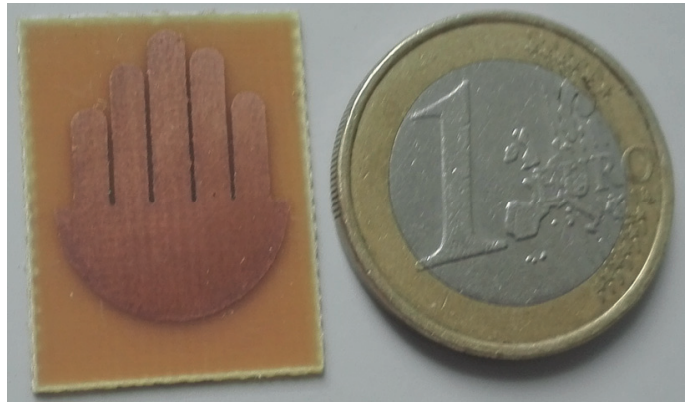

Fig. 3. Photo of the fabricated tag.

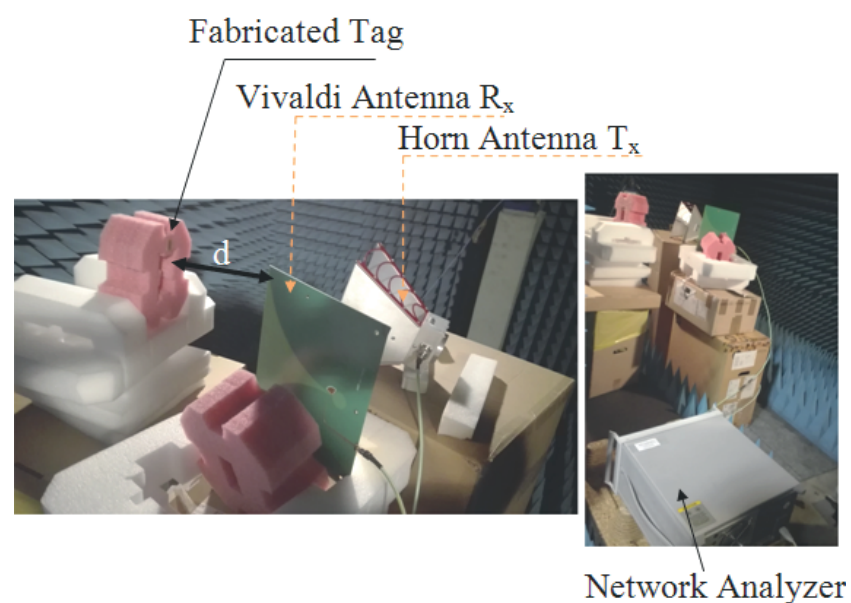

Fig. 4. The measurement setup for our slotted hand-based chipless tag.

from $10 \mathrm{MHz}$ to $67 \mathrm{GHz}$. The two VNA ports are connected to a $T_{x}$ horn antenna and an $R_{x}$ Vivaldi antenna, respectively. These antennas are positioned in front of the tag under test with a distance of $d=20 \mathrm{~cm}$ and separated from each other with a distance of $10 \mathrm{~cm}$ as shown in Fig. 4. The antennas are well operating in the whole operating band of the chipless tag. Using this set-up, the RCS response is measured.

As shown in Fig. 5, there are four resonant frequencies in the selected operating band. Besides, the last measured resonant peak shows a slight shift compared to the simulated response because of the coupling between $\mathrm{Tx}$ and Rx antennas. Also, for reasons of cable losses, the last peak is deformed and we notice the appearance of a parasitic peak near the fourth peak. Similarly, the changes in material parameters can have a significant impact on the measurement results in our case.

Typical RF and microwave materials have a dielectric constant with a tolerance of less than $3 \%$ at $10 \mathrm{GHz}$, a dimensional stability of less than $5 \%$, loss tangent values of less than 0.005 which are required for most applications. The FR4 material has a typical loss tangent in the region of 0.02 , which is too high for many circuit applications, and stability is, however, very good. Consequently, the use of FR4 for microwave circuits is not likely to be viable for electrically large antenna designs due to its high losses. However, for broadband antenna elements, where losses and

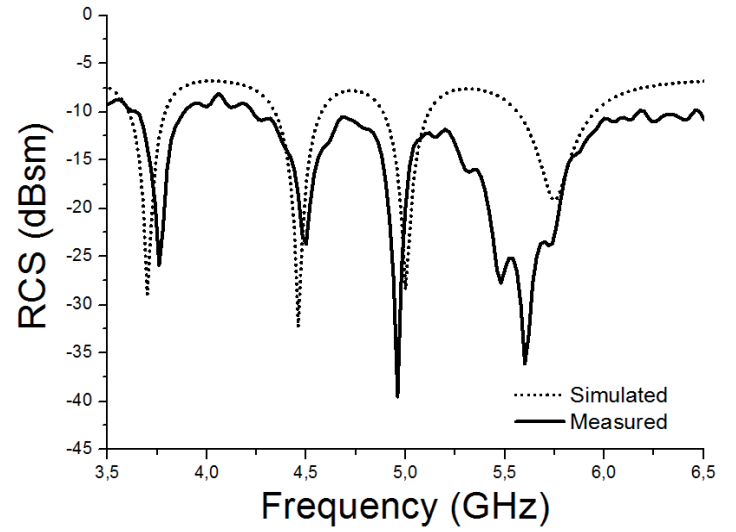

Fig. 5. Superposition of simulated and measured RCS of the chipless tag.

absolute dielectric constant values are less critical, the material can be used in place of more conventional microwave substrates, offering significant cost savings [22].

\subsection{Encoding Approach}

Our purpose is to choose the chipless tag encoding approach.

The electric field distribution in the resonant slotted hand-based tag proves successfully that the four slots are the origin of the four resonances shown in the operating band. This leads to basically use the slots for the selected encoding approach. Consequently, and according to the multi-frequency response, we can deduce that the frequency domain approach will be adopted to enable an increase in the coding capacity. So, instead of coding by the absence or presence of the resonant frequencies, the coding technique consists of shifting the different frequencies. Adding a metal strip in different positions between the fingers mainly within the slots was proposed in order to try to shift frequencies.

In fact, inserting a metal strip between the first and second finger can limit the radiation in the first slot, which can shift the frequency. So, a new encoding binary state is obtained. The shift can affect the first, third and fourth frequency as proves the E-field distribution study within the slot at the three frequencies.

Figure 6 shows the manner of inserting a metal strip, which has a length and width of $0.2 \mathrm{~mm}$, in the same $\mathrm{y}$-position in the different slots. It gives the RCS responses. While the first position is represented when inserting the metal strip in the first slots shown in Fig. 6(a), the second position is obtained when placing the strip in the second slot as Fig. 6(b) shows. Added to that, the third position is marked by the insertion of the metal strip within the third slot (see Fig. 6(c), and Figure 6(d) clearly shows its fourth position. Thus, thanks to this technique of frequency shifting, it is possible to enhance the data density and to double the coding capacity by using bit combinations ensuring a data of 8-bit capacity [12]. 

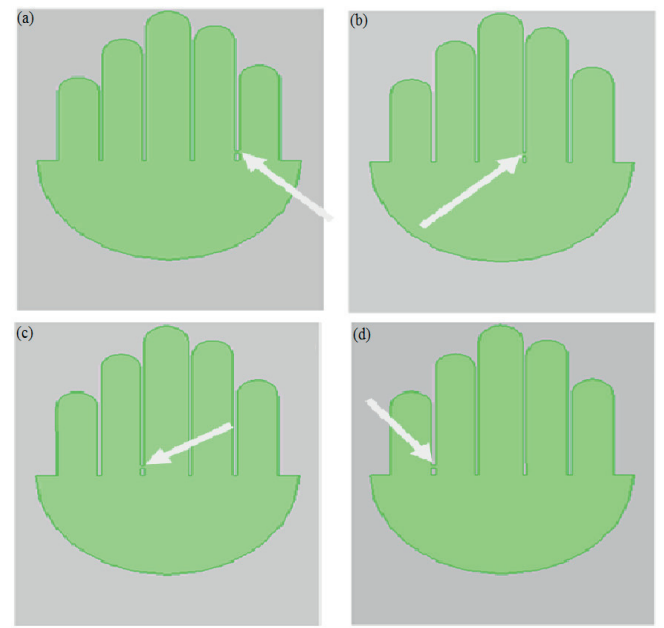

Fig. 6. The encoding approach by inserting a metal strip: (a) the metal strip placed at the first slot, (b) the metal strip at the second slot, (c) the metal strip at the third slot, (d) the metal strip at the fourth slot.

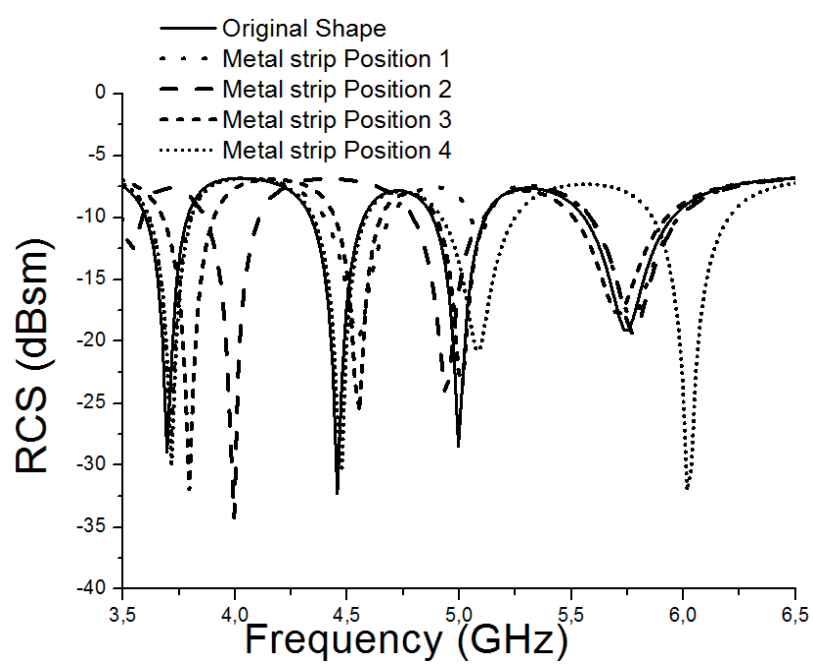

Fig. 7. Superposition of RCS responses of the four configurations (influence of adding a metal strip).

The concept behind this technique can be described as follows: a single resonant frequency is used to generate four binary states by changing the position of the metal strip along the slot. The metal strip position which shifts the frequency to a lower value represents the bit combination ' 10 ', and the metal strip position which shifts the frequency to a higher value gives the bit combination ' 01 '. If the original frequency is still unchangeable, we affect the bit combination ' 11 ', and if the response gives an exceeded value of the tag operating band or realizes a frequency shift greater than $500 \mathrm{MHz}$, we affect the bit combination '00'.

Table 2 details the different frequency shifts obtained in the four conditions. Moreover, to validate the adopted encoding approach, the simulation response of placing the metal strip in the third position is compared to the main shape which is taken as a reference as shown in Fig. 8.

This results in a binary state composed of 8 bits. Mainly, the shift of the first frequency to a higher value of $3.8 \mathrm{GHz}$ leads to assign ' 01 ' combination in this position.

\begin{tabular}{|c|c|c|c|c|}
\hline $\begin{array}{c}\text { Original } \\
\text { frequency } \\
(\mathbf{G H z})\end{array}$ & $\begin{array}{c}\text { Frequencies } \\
{\text { of the } \mathbf{1}^{\text {st }}}^{\text {metal strip }} \\
\text { position } \\
(\mathbf{G H z})\end{array}$ & $\begin{array}{c}\text { Frequencies } \\
\text { of the } \mathbf{2}^{\text {nd }} \\
\text { metal strip } \\
\text { position } \\
(\mathbf{G H z})\end{array}$ & $\begin{array}{c}\text { Frequencies } \\
{\text { of the } \mathbf{3}^{\text {rd }}}^{\text {metal strip }} \\
\text { position } \\
(\mathbf{G H z})\end{array}$ & $\begin{array}{c}\text { Frequencies } \\
{\text { of the } \mathbf{4}^{\text {th }}}^{\text {metal strip }} \\
\text { position } \\
(\mathbf{G H z})\end{array}$ \\
\hline 3.7 & 3.72 & 3.54 & 3.8 & 3.72 \\
\hline 4.46 & 4.56 & 4 & 4.56 & 4.48 \\
\hline 5 & 5.1 & 4.94 & 5 & 5.1 \\
\hline 5.75 & 5.75 & 5.78 & 5.72 & 6.02 \\
\hline
\end{tabular}

Tab. 2. Frequency shifts values for four positions of the metal strip.

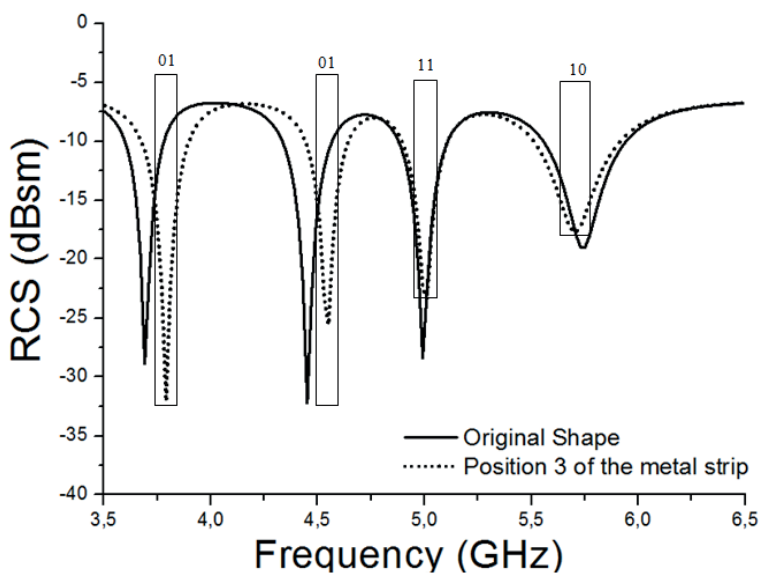

Fig. 8. Superposition of two encoding states.

Also, the second frequency gives a shift to a higher value of $4.56 \mathrm{GHz}$, which allows to affect the same previous ' 01 ' combination in this position. The third frequency is still unchangeable, which leads to attribute the ' 11 ' combination to this value. The fourth frequency ensures a shift to a lower value of $5.72 \mathrm{GHz}$, giving the ' 10 ' binary combination. As a result, the obtained binary state is represented by '01011110'.

\subsection{Permittivity Monitoring Based on RFID Chipless Tag Sensor}

For our tag sensor, the dielectric properties of a material which is characterized by an arbitrary shape and geometry are detected. Thus, using the same design of our proposed chipless tag, this section presents a permittivity sensor. It denotes a new application for chipless tags. A passive radio frequency tag is attached to an object to estimate its electrical properties and to monitor its evolution over time, or after use. Note that the change in electrical properties can be correlated with the level of Relative Humidity (RH) [21]. As, the mechanism for permittivity sensing used here is based on frequency shifting. We present the simulated performance of the sensing tag with phantoms of varying permittivity.

Hence, permittivity measurement of materials plays a very important role in several industrial, scientific, and medical applications. Diverse techniques exist for the measurement of permittivity of low loss dielectrics, as resonant methods and transmission or reflection methods. The permittivity of materials can be determined from the 
measured changes of the resonance frequency and quality factor of a resonant cavity [23-25]. The objective of this study is to develop a low-cost system permittivity sensor in the ultra-wideband frequency range.

Therefore, statistical studies have proven that the relative permittivity of any substrate varies significantly with frequency, with fiberglass and with resin contents, which is an important topic and has a significant impact on measurement results if substantial changes in the material parameters are shown. So, if the necessary information on materials parameters is inaccessible in knowledge bases, the relative permittivity at the assumed frequency band should be determined experimentally using some methods as $\mathrm{T}$ and modified ring resonator technique detailed in [26], or also using this proposed method.

Moreover, the chipless tag has been used to realize a passive wireless permittivity sensor. Its layout is shown in Fig. 1(B). The material under test was placed on the top of the hand-shaped tag forming a superstrate. Thus, it resonates at a frequency determined by its equivalent shape. A simulation was developed to investigate the ability of the proposed sensor to give an independent response added to the response of the RFID chipless tag. The first tested substrate is the polystyrene with a dielectric permittivity of 2.6, and a thickness $h=0.00019 \mathrm{~mm}$.

As shown in Fig. 9, a new resonant peak is appeared at $6.86 \mathrm{GHz}$. It is the sensing response. As a comparison with the tag response shown in Fig. 5, it is clearly seen that the four resonant peaks of identification are not changed, and the apparition of the new peak is related to the material placed on the tag.

Thus, and as described previously, the addition of the strip at a defined position shifts the frequency and gives a new binary state. That's why, the addition of the material to be characterized must not influence the main role of identification, and it should not be placed on the identification surface.

Furthermore, additional material tests have been performed. A summary of the simulated frequency shifts is

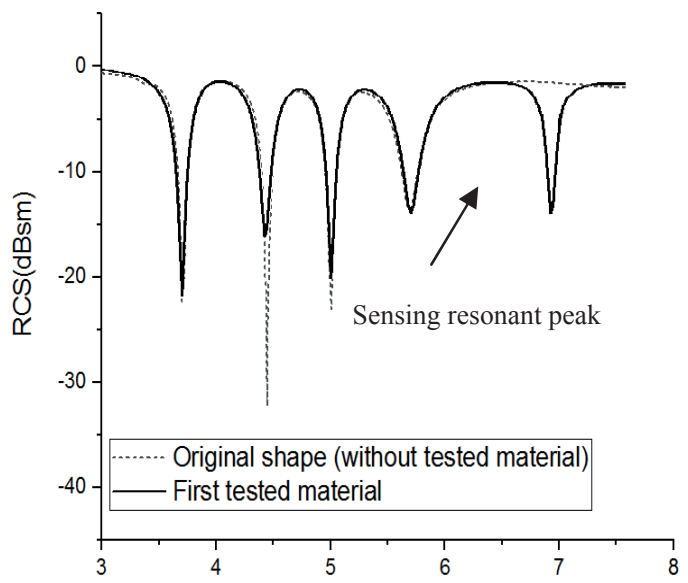

Fig. 9. The simulated response of the permittivity sensor (tested material: polystyrene).

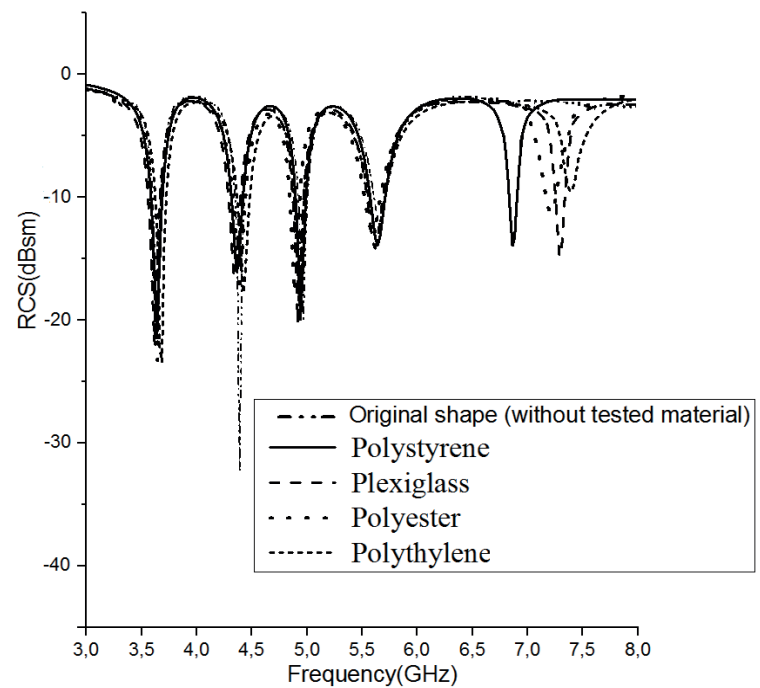

Fig. 10. Superposition of the tested sensed materials.

\begin{tabular}{|c|c|c|}
\hline Material [27] & $\varepsilon_{\mathbf{r}}$ & Frequency [GHz] \\
\hline Polystyrene & 2.6 & 6.86 \\
\hline Plexiglass & 3.4 & 7.34 \\
\hline Polyester & 3.2 & 7.28 \\
\hline Polythylene & 2.25 & 7.42 \\
\hline Taconic TLX-0 & 2.45 & 7.18 \\
\hline Rogers RO4003 & 3.55 & 7.22 \\
\hline
\end{tabular}

Tab. 3. Frequency shifts values for tested materials.

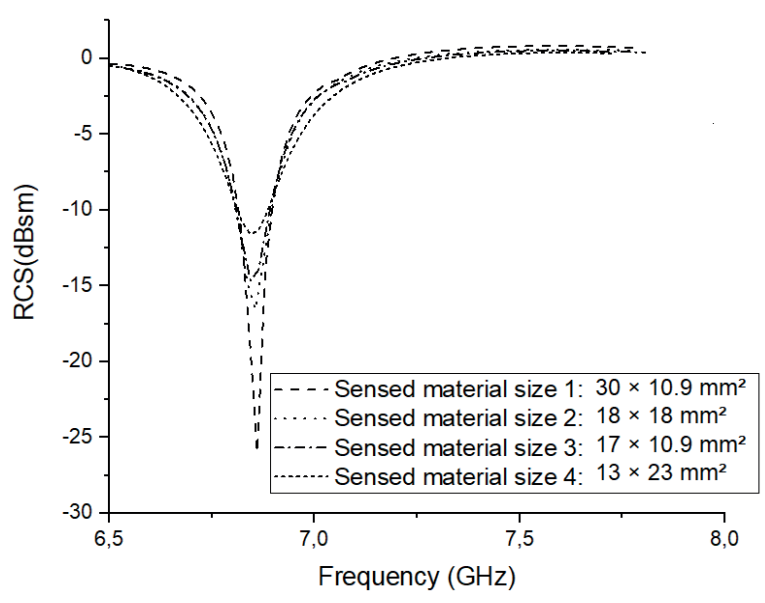

Fig. 11. Superposition of 4 different sizes of polystyrene.

reported in Tab. 3. Figure 10 shows the operating band of the sensor where it is clearly seen that resonant peak's position of the identification band is unchanged, and a sensing band is appeared from 6.5 to $8 \mathrm{GHz}$ characterized by a new frequency position for each different dielectric constant.

We need to mention that the size of the sensed material influences the adaptation of the sensing resonant peak as Figure 11 shows.

As well, some tests of the size and position of the tested objects are achieved in order to prove the feasibility of the proposed RFID chipless tag sensor. Therefore, the 


\begin{tabular}{|c|c|c|c|c|c|}
\hline $\begin{array}{c}\text { Chipless tag for sensing } \\
\text { application }\end{array}$ & {$[\mathbf{2 8}]$} & {$[\mathbf{1 5}]$} & {$[\mathbf{1 6 ]}$} & {$[\mathbf{1 7 ]}$} & $\begin{array}{c}\text { The proposed sensitive } \\
\text { chipless tag }\end{array}$ \\
\hline Tag size $\left(\mathbf{m m}^{\mathbf{2}}\right)$ & $90.5 \times 55.9$ & $36 \times 26$ & $\begin{array}{c}2 \times 2 \text { cells } \\
1 \text { cell }=22 \times 20\end{array}$ & $46.2 \times 25$ & $23 \times 17.56$ \\
\hline Encoding principle & $\begin{array}{c}\text { Spectrogram } \\
\text { method }\end{array}$ & $\begin{array}{c}\text { Notch frequency and } \\
\text { notch amplitude } \\
\text { position }\end{array}$ & Frequency shifting & $\begin{array}{c}\text { Frequency } \\
\text { shifting }\end{array}$ & Frequency shifting \\
\hline Operation band (GHz) & $2-8$ & $2.6-2.9$ & $3-4.5$ & $3.5-8$ & $\begin{array}{c}3.5-6.5: \text { Identification } \\
\text { band } \\
6.5-8: \text { sensing band }\end{array}$ \\
\hline Substrate thickness $(\mathbf{m m})$ & 10 & 0.043 & 0.119 & 0.8 & 0.8 \\
\hline
\end{tabular}

Tab. 4. Comparative analysis between the proposed chipless tag and three prototypes from the existing literature.

arbitrary size of the tested object influences the adaptation of the sensing resonant peak without impacting its position as seen in Fig. 11. A reduced size of the sensed material polystyrene decreases the adaptation of the resonant peak, and each increase in size gradually improves its RCS response. Consequently, chipless tag using high performance substrate is under research in order to improve the sensor acting.

Hence, the simulation results prove that the proposed sensor can be a low-cost substitute to measure the permittivity of a material with arbitrary dimensions and offers the advantages of a low weight and an original shape based on a chipless tag which uses a classic substrate FR4.

\subsection{Comparative Analysis}

Table 4 shows the comparison of the proposed RFID sensing chipless tag with some successful designs from literature. The proposed prototype appeared as an interesting contribution. It offers the advantages of a reduced size, a simple layer, and an original shape guaranteeing an important encoding capacity of identification with permittivity monitoring of specific sizes material. It can be seen that improved results are presented using the frequency shifting technique in the identification and sensing bands. The utilization of FR4 substrate yields good results as well as good agreement between measurement and simulation. Hence, the sensing tag tests confirm the choice of tag shape and parameters as well as the acquired performances that clearly show an innovative contribution to the chipless technology.

\section{Conclusion}

The strong motivation behind this work is to achieve the identification task and to represent the permittivity of material over time or after use. We have developed a compact RFID chipless tag using multiple slots etched on a hand-shaped tag. The first proposed design has the potential of giving 8-bits as data capacity with a reduced size by simply etching 4 slots on the shape of the hand, and adopting a frequency shifting technique. Further, the metal slotted hand was used to put an additional slot in order to realize a passive wireless permittivity sensor. Future configura- tions will contribute to simplify its printability on objects using high performance substrate.

\section{References}

[1] PRERADOVIC, S., KARMAKAR, N, C. Chipless RFID: Bar code of the future. IEEE Microwave Magazine, 2010, vol. 11, no. 7, p. 87-97. DOI: 10.1109/MMM.2010.938571

[2] HARTMANN, C. S. A global SAW ID tag with large data capacity. In Proceedings of the IEEE Ultrasonics Symposium. Munich (Germany), 2002, p. 65-69. DOI: 10.1109/ULTSYM.2002.1193354

[3] HARTMANN, C. S., BROWN, P., BELlAMY, J. Design of global SAW RFID tag devices. In Proceedings of the Second International Symposium on Acoustic Wave Devices for Future Mobile Communication Systems. Chiba University (Japan), March 2004, p. 1-5.

[4] COSTA, F., GENOVESI, S., MONORCHIO, A. Chipless RFIDs for metallic objects by using cross polarization encoding. IEEE Transactions on Antennas and Propagation, 2014, vol. 62, no. 8, p. 4402-4407. DOI: 10.1109/TAP.2014.2326421

[5] PRERADOVIC, S., ROY, S. M., KARMAKAR, N. C. RFID system based on fully printable chipless tag for paper-/plastic-item tagging. IEEE Antennas and Propagation Magazine, 2011, vol. 53, no. 5, p. 15-32. DOI: 10.1109/MAP.2011.6138421

[6] JALALY, I., ROBERTSON, I. D. RF barcodes using multiple frequency bands. In IEEE MTT-S International Microwave Symposium Digest. Long Beach (CA, USA), 2005, p. 139-141. DOI: $10.1109 /$ MWSYM.2005.1516542

[7] KALANSURIYA, P., KARMAKAR, N., VITERBO, E. On the detection of frequency-spectra-based chipless RFID using UWB impulsed interrogation. IEEE Transactions on Microwave Theory and Techniques, 2012, vol. 60, no. 12, p. 4187-4197. DOI: 10.1109/TMTT.2012.2222920

[8] ATTARAN, A., RASHIDZADEH, R. Chipless radio frequency identification tag for IoT applications. IEEE Internet of Things Journal, 2016, vol. 3, no. 6, p. 1310-1318. DOI: 10.1109/JIOT.2016.2589928

[9] REZAIESARLAK, R., MANTEGHI, M. A space-time-frequency anticollision algorithm for identifying chipless RFID tags. IEEE Transactions on Antennas and Propagation, 2014, vol. 62, no. 3, p. 1425-1432. DOI: 10.1109/TAP.2013.2295393

[10] FENG, C., ZHANG, W., LI, L., et al. Angle-based chipless RFID tag with high capacity and insensitivity to polarization. IEEE Transactions on Antennas and Propagation, 2015, vol. 63, no. 4, p. 1789-1797. DOI: 10.1109/TAP.2015.2393851 
[11] SAKOUHI, S., RAgGad, H., GHARASAllah, A., et al. A novel RFID EMSICC-based chipless tag. Radioengineering, 2017 , vol. 26 , no. 1 , p. $154-161$. DOI: $10.13164 /$ re.2017.0154

[12] SAKOUHI, S., RAGAD, H., LATRACH, M. Development of a novel radio frequency identification chipless tag with multifrequency response. International Journal of $R F$ and Microwave Computer-Aided Engineering, 2019, vol. 29, no. 11, p. $1-8$. DOI: $10.1002 /$ mmce. 21735

[13] JAVED, N., HABIB, A., AMIN, Y., et al. Miniaturized flexible chipless RFID tag for IoT market. In 2017 International Conference on Communication, Computing and Digital Systems (C-CODE). Islamabad (Pakistan), 2017, p. 71-74. DOI: 10.1109/C-CODE.2017.7918904

[14] SALMERÓN, J., ALBRECHT, A., KAFFAH, S., et al. Wireless chipless system for humidity sensing. Sensors, 2018, vol. 18, no. 7, p. 1-11. DOI: $10.3390 / \mathrm{s} 18072275$

[15] WILTSHIRE, B. D., ZARIFI, T., ZARIFI, M. H. Passive split ring resonator tag configuration for RFID-based wireless permittivity sensing. IEEE Sensors Journal, 2019, vol. 20, no. 4, p. 1904 to 1911. DOI: $10.1109 /$ jsen.2019.2950912

[16] LAZARO, A., VILlarino, R., COSTA, F., et al. Chipless dielectric constant sensor for structural health testing. IEEE Sensors Journal, 2018, vol. 18, no. 13, p. 5576-5585 DOI: 10.1109/JSEN.2018.2839689

[17] SAKOUHI, S., RAGGAD, H., GHARSALlaH, A., et al. A quarter mode substrate integrated circular cavity chipless tag based humidity sensor. In IEEE 19th Mediterranean Microwave Symposium (MMS). Hammamet (Tunisia), 2019, p. 1-5. DOI: 10.1109/MMS48040.2019.9157294

[18] MARINDRA, A. M. J., TIAN, G. Y. Multiresonance chipless RFID sensor tag for metal defect characterization using principal component analysis. IEEE Sensors Journal, 2019, vol. 19, no. 18, p. $8037-8046$. DOI: $10.1109 /$ jsen.2019.2917840

[19] SAKOUHI, S., RAGGAD, H., GHARSALlaH, A., et al. Design of five-shaped fingers based RFID chipless tag. In 2019 Antennas Design and Measurement International Conference (ADMInC). St. Petersburg (Russia), 2019, p. 23-25. DOI: 10.1109/ADMInC47948.2019.8969351

[20] DISSANAYAKE, T., ESSELlE, K. P. Prediction of the notch frequency of slot loaded printed UWB antennas. IEEE Transactions on Antennas and Propagation, 2007, vol. 55, no. 12, p. 3320-3325. DOI: 10.1109/TAP.2007.908792

[21] MIACCI, M. A. S., REZENDE, M. C. Basics on radar cross section reduction measurements of simple and complex targets using microwave absorbers. In Haq, M. D. (ed.) Applied Measurement Systems, 2012, Ch. 16, p. 361-389. DOI: $10.5772 / 37195$

[22] AgUilar, J. R., BEADLE, M., THOMPSON, P. T., et al. The microwave and RF characteristics of FR4 substrates. In IEE Colloquium on Low Cost Antenna Technology. London (UK), 1998, p. 1-6. DOI: 10.1049/ic:19980078
[23] CHEN, L. F., ONG, C. K., NEO, C. P., et al. Microwave Electronics: Measurement and Materials Characterization. $1^{\text {st }}$ ed. New York (USA): John Wiley \& Sons, 2004, p. 37-90. ISBN: 978-0470844922

[24] SUBRAMANIAN, V., SIVASUBRAMANIAN, V., MURTHY, V. R. K., et al. Measurement of complex dielectric permittivity of partially inserted samples in a cavity perturbation technique. Review of Scientific Instruments, 1996, vol. 67, no. 1, p. 279-282. DOI: $10.1063 / 1.1146548$

[25] LOBATO-MORALES, H., CORONA-CHÁVEZ, A., MURTHY, D. V. B., et al. Complex permittivity measurements using cavity perturbation technique with substrate integrated waveguide cavities. Review of Scientific Instruments, 2010, vol. 81, p. 1-4. DOI: $10.1063 / 1.3442512$

[26] JANKOWSKI-MIHUŁOWICZ, P., LICHOŃ, P., PITERA, G. W., et al. Determination of the material relative permittivity in the UHF band by using $\mathrm{T}$ and modified ring resonators. International Journal of Electronics and Telecommunications, 2016, vol. 62, no. 2, p. 129-134. DOI: 10.1515/eletel-2016-0017

[27] https://laminatedplastics.com/alpheticalist.php

[28] ALI, Z., PERRET, E., BARBOT, N., et al. Extraction of aspectindependent parameters using spectrogram method for chipless frequency-coded RFID. IEEE Sensors Journal, 2021, vol. 21, no. 5, p. 6530-6542. DOI: 10.1109/jsen.2020.3041574

\section{About the Authors ...}

Soumaya SAKOUHI received the degree in Electrical Engineering in 2013, and the Ph.D. degree in Electrical Engineering from the Faculty of Sciences of Tunis, Tunisia in 2019. Her main research interests include RFID and SIW technology.

Hedi RAGGAD received his Ph.D. degree from ESEO, Angers, France. His research interests include dielectric resonator antennas.

Ali GHARSALLAH is a Full Professor in Electrical Engineering at the Faculty of Sciences of Tunis, Tunisia. His current research interests include smart antennas, multilayered structures and integrated circuits.

Mohamed LATRACH is a Professor of Microwave Engineering and head of the Radio \& Microwave group at ESEO, Angers, France. His field of research interest is the design of microwave circuits. 